\title{
Involvement of pro-apoptotic and pro-autophagic pro- teins in Granulosa cell death
}

\author{
EscobarML, EcheverríaOM, CasasaAS, García G, AguilarSJ, Vázquez-NinGH* \\ Laboratorio de Microscopía Electrónica. Departamento de Biología Celular. Facultad de Ciencias. Universidad Nacional Autónoma de \\ México (UNAM).
}

\section{Email address:}

vazqueznin@ciencias.unam.mx(V-NinGH)

\section{To cite this article:}

Escobar M L, Echeverría O M;Casasa A S, García G, Aguilar S J, Vázquez-NinGH. Involvement of Pro-Apoptotic and Pro-Autophagic Proteins in Granulosa Cell Death Cell Biology Vol. 1, No. 1, 2013, pp. 9-17. doi: 10.11648/j.cb.20130101.12

\begin{abstract}
Follicular atresia is a process present in all mammals studied. It involves the oocyte and granulosa cells. The apoptotic cell death has been implicated in follicular atresia. Now it is known that the autophagy is a programmed cell death. In this work, atretic follicles of Wistar rats' ovaries were analyzed, to evaluate the routes of granulosa cell death during the follicular atresia. The apoptosis and autophagy presence was studied by means of ultrastructural and immunohistochemical techniques, and by molecular procedures. During atresia, follicular cells undergo the standard processes of cell death, apoptosis and autophagy, as well as a process in which features of both occur in the same cell. Other processes of cell death affect only granulosa cells and involve such features as contraction of cell volume, an increase of the lumen of the nuclear envelope and the endoplasmic reticulum, and loss of contacts with the oocyte, which is also altered.
\end{abstract}

Keywords: Atresia, Apoptosis, Autophagy, Granulosa Cell

\section{Introduction}

The follicle is a morphological and physiological unit that can remain unchanged, may grow into an ovulating follicle, or may suffer regressive changes called atresia. Follicular atresia is the process through which ovarian follicles not selected for ovulation are eliminated. Ovarian follicles in the process of atresia occur frequently in the ovaries of newborn, prepubertal and adult female mammals. The eventual fate of the follicle depends on a balance in the expression and actions of the factors that promote the proliferation, growth and differentiation of follicular cells, and those that induce programmed cell death. FSH binds to its granulosa cell receptors to promote the survival and growth of ovarian follicles by stimulating proliferation and estradiol secretion and inhibiting cell death by up-regulating the expression of anti-apoptotic proteins [1].

During follicular atresia, the oocyte and granulosa cells undergo processes of cell death. In primordial follicles, the onset of the process of atresia seems to depend on alterations of the oocyte[2]. Immunohistochemical and ultrastructural analyses of the processes of cell death in oocytes in newborn and prepubertal female rats have demonstrated the presence of abundant autophagosomes, apoptotic bodies, a positive reaction to the TUNEL method, active caspase-3 and Lamp-1, and the absence of large clumps of compact chromatin associated with the nuclear membrane [3]. In adult rats, markers of both apoptosis and autophagy were found in the same oocyte [4]. The structural features of the process of oocyte death are characterized by the presence of clear vacuoles and autophagosomes, and the absence of large clumps of compact chromatin and apoptotic bodies [5, $6]$. The main cytochemical feature of the oocytes was their positive reaction to the TUNEL method, which changed according to the age of the rats studied [3].

The apoptotic process of cell death (programmed cell death type I) has been implicated in follicular atresia, as several studies have shown the different morphological characteristics of that process with, and the expression of several proteins related to the apoptotic cell death process has been identified in granulosa cells during follicular progress [7]. Apoptotic cell death is carried out by proteases called caspases; enzymes that are classified into two groups: initiator caspases (such as 8 and 9), and effector caspases $(3,6,7)$. Once activated, these proteases cleave to different cellular proteins [8].

Programmed cell death process type II -autophagy- is characterized morphologically by the presence of a large 
number of autophagosomes with cytoplasmic content in different degrees of degradation [9].

Molecular mechanisms involved in autophagy suggest the participation of atg genes [10]. In mammals, the microtubule-associated protein 1 light chain 3 (LC3), the Atg8 homologue, has been used as an indicator of autophagy. The phosphatidylethanolamine (PE)-conjugated Atg8 protein plays a structural role in autophagy and is a useful tool for evaluating that process [11]. Beclin-1 (Atg6) is another autophagy-related protein with a fundamental role in autophagy [12], it is involved in the initiation of the autophagy $[13,14]$, in the formation [15] and maturation [16] of autophagosomes.

The aim of the present study is to analyze the structural, ultrastructural, cytochemical, and molecular aspects of granulosa cell alterations together with modifications of the interrelations among rat granulosa cells and between them and the oocyte.

\section{Materials and Methods}

\subsection{Experimental animals}

60 female Wistar rats were used in the study. They were kept under a $12 \mathrm{~h}$ light-darkness cycle with water and food ad libitum. All animals were handled in accordance with the NIH Guide for the Care and Use of Laboratory Animals.

\subsection{Ovary tissue processing for optical microscopy}

Ovaries were processed as described elsewhere [4]. They were fixed in $2 \%$ paraformaldehyde in phosphate-buffered saline (PBS) at $\mathrm{pH} 7.2$ for 2 hours at room temperature, embedded in paraffin, and serially sectioned. Five $\mu \mathrm{m}$-thick sections from each ovary were aligned on glass microscope slides covered with poli-l-lysine (Sigma, St Louis, MO), and observed under a Nikon Eclipse E600 Microscope. Images were recorded with a Nikon Digital Camera DXM1200F.

\subsection{Electron microscopy process}

Ovaries were fixed in $2.5 \%$ glutaraldehyde- $4 \%$ formaldehyde in phosphate-buffered saline (PBS) at $\mathrm{pH} 7.2$ for 2 hours. After rinsing in the same buffer, tissues were postfixed in $1 \%$ osmium tetroxide (OsO4) in a PBS buffer at $\mathrm{pH} 7.2$ for 1 hour. Tissues were then dehydrated using a graded ethanol series and embedded in Epon (Embed-812, Electron Microscopy Science; Hatfield, PA). Semi-thin sections were stained with toluidine blue. Selected areas were thin-sectioned and mounted on grids, which were counterstained with uranyl acetate and lead citrate. Sections were examined under a JEOL 1010 electron microscope operated at $100 \mathrm{KV}$. Digital images were taken with a Hamamatsu camera.

\subsection{Immunodetection assays}

Ovaries were fixed in $4 \%$ formaldehyde in PBS at $\mathrm{pH}$ 7.2 for 2 hours. The tissues were dehydrated using a graded ethanol series and embedded in paraffin. The paraffinembedded samples were then sectioned for use in carrying out immunolocalizations of the active caspase-3, Beclin-1, and LC3 proteins, and to perform the TUNEL technique. Antigen unmasking was carried out by microwaving the tissue sections in citrate-buffer at $0.1 \mathrm{M}$ and $\mathrm{pH} 6$ (BioGenex, Fremont, CA) in a Panasonic microwave oven for 3 min at $1,300 \mathrm{~W}$, and then for $6 \mathrm{~min}$ at $780 \mathrm{~W}$. After cooling, the sections were washed in PBS and incubated with primary antibodies.

\subsection{Simultaneous Beclin-1 and active caspase-3 immuno- localization}

Deparaffinized tissue sections were washed in PBS and incubated simultaneously with anti-active caspase-3 (Sigma, St Louis, MO) and anti-Beclin-1 (Abcam, Cambridge, UK) (1:100) in PBS dilution for $18 \mathrm{~h}$ at $4^{\circ} \mathrm{C}$. Negative controls were elaborated by omitting the primary antibodies. After washing, the slides were incubated for $1 \mathrm{~h}$ under darkness at room temperature with an anti-rabbit immunoglobulin coupled to Alexafluor488(Invitrogen Carlsbad, CA) and anti-sheep immunoglobulin that in turn was coupled to Alexafluor 594 (Invitrogen; Carlsbad, CA). Next, the preparations were washed and counterstained with DAPI (Sigma, St Louis, MO) to evaluate DNA distribution. Slides were covered with mounting media for fluorescence microscopy (Vectashield Mounting Medium, Vector Labs., Burlingame, CA).

\subsection{Simultaneous TUNEL procedure and LC3 immuno- detection}

Deparaffinized tissue sections were washed in PBS and simultaneously incubated with primary antibody anti-LC3 (Affinity BioReagents; Rockford, IL) (1:200) in PBS dilution for $18 \mathrm{~h}$ at $4^{\circ} \mathrm{C}$. After washing, slides were incubated with an anti-rabbit immunoglobulin coupled to Alexafluor 488 (Invitrogen; Carlsbad, CA) for $1 \mathrm{~h}$ under darkness at room temperature. Then the preparations were washed and the evaluation of DNA fragmentation was performed using the Apoptag Red In Situ Apoptosis Detection Kit (Millipore; Co, USA). Sections were labeled with biotin-dUTP by incubation with a reaction buffer containing terminal deoxinucleotidyltransferase for $1 \mathrm{~h}$ at $37^{\circ} \mathrm{C}$. Biotinylated nucleotides were detected with a streptavidin-Rhodamine conjugate. Tissues were counterstained with DAPI (Sigma, St Louis, MO) to evaluate DNA distribution. Slides were covered with Vectashield Mounting Medium for fluorescence microscopy. The negative controls were carried out omitting the primary antibody or the terminal deoxinucleotidyltransferase. 


\subsection{Annexin-V assay}

The apoptotic granulosa cells were identified by fluorescence microscopy using the Annexin-V/FITC Apoptosis Detection Kit (Sigma, St Louis, MO) according to the manufacturer's instructions. $5 \mu \mathrm{l}$ of Annexin-V/FITC and 1 $\mu \mathrm{l}$ of propidium iodide to the cellular fraction were added. Then the preparation was incubated at $37^{\circ} \mathrm{C}$, protected from light, for exactly $10 \mathrm{~min}$. The cells in early apoptotic process were stained by the Annexin-V/FITC alone.

\subsection{Western Blot analysis}

The protein expression level was evaluated using the Western Blot assay. The granulosa cells were isolated from the ovaries of the Wistar rats, which were disaggregated in $0.1 \%$ trypsin (GIBCO; Grand Island, NY) in a calcium- and magnesium-free solution for $15 \mathrm{~min}$ at $37^{\circ} \mathrm{C}$. The ovarian cells were then incubated at $37^{\circ} \mathrm{C}$ in an atmosphere containing $5 \% \mathrm{CO} 2$ for $24 \mathrm{~h}$ on $35 \mathrm{~mm}$ culture plates (Nunc; Denmark), with DMEM GlutaMAX(GIBCO; Grand Island, NY) supplemented with $0.1 \%$ albumin (Sigma, St Louis, $\mathrm{MO}$ ) and 4\% fetal bovine serum (GIBCO; Grand Island, NY). The cells were incubated for $24 \mathrm{~h}$ to allow the granulosa cells to adhere to the bottom of the dish. Next, the dishes were washed and the granulosa cells were incubated for $15 \mathrm{~min}$ in lysis buffer (50 mMTris- $\mathrm{Cl}, \mathrm{pH} 7.5 ; 150$ $\mathrm{mMNaCl}, 0.1 \%$ SDS, $1 \mathrm{mM}$ PMSF, $0.5 \%$ sodium deoxycholate, and $1 \%$ Nonidet P-40), supplemented with the complete protease inhibitor cocktail (Roche, Mannheim, Germany). Total proteins were measured by Bradford Assay: $50 \mu \mathrm{g}$ of total proteins were loaded onto a $12 \%$ SDSPAGE gel before being transferred to polyvinylidene fluoride (PVDF) membranes, which were incubated for $1 \mathrm{~h}$ at room temperature in blocking buffer. Then the membranes were incubated with anti-active caspase- 3 and antiLC3 antibodies at a dilution of 1:5000. Afterwards, the proteins were tagged by incubation with peroxidaseconjugated secondary antibody (Jackson, Newmarket, UK) at 1:10,000 in blocking buffer for $1 \mathrm{~h}$ at room temperature. Using Horse Radish Peroxidase (HRP) as the substrate (Immobilon Western, Millipore Co, USA), specific labeling was detected by chemiluminescence. Amersham Biosciences Hyperfilm(Amersham Biosciences, UK) was exposed to the membranes to detect chemiluminescence.

\subsection{DNA fragmentation by electrophoresis}

The presence of a ladder pattern of DNA degradation during the apoptotic process of cell death was evaluated by electrophoresis of ovarian DNA, which was obtained from the rats' ovaries using DNAzol (Invitrogen; Carlsbad, CA), following the manufacturer's instructions. DNA electrophoresis was carried out in a $2 \%$ agarose gel and visualized by ethidium bromide.

\subsection{0. $m R N A$ by reverse transcriptase-polymerase chain reaction ( $R T-P C R)$}

Total RNA from the rats' ovaries was prepared using Trizol (Invitrogen; Carlsbad, CA), following the manufacturer's instructions. RT-PCR analysis was performed in a Mastercycler gradient (Eppendorf, Germany). For cDNA synthesis, OligodT (Applied Biosystem) was used as a primer following the condition for Transcriptor Reverse Transcriptase (Roche; Mannheim, Germany). The PCR contained $1 \mathrm{X}$ Taq polymerase buffer, $0.2 \mu \mathrm{M}$ each of dNTPs, $0.5 \mathrm{ml}$ of Taq Polymerase (Roche Applied Science; New Jersey), and $20 \mathrm{mM}$ of the following primers: Caspase-3 Fwd GCCTGTCCTGGATAAGACCA, Rev TTGACTCAGAAGCCGAAGGT; LC3 Fwd TGGCCCTGAAATACGAAGTC, Rev GGCAGTAGTCGCCTCTGAAG; $\quad \beta$-Actin $\quad$ Fwd GTATGCCTCTGGTCGTACCA, and Rev CTTCTGCATCCTGTCAGCAA, as reported previously (6). The conditions for PCR were as follows: 2 min of initial denaturation at $94^{\circ} \mathrm{C}$ for 32 cycles $\left(94^{\circ} \mathrm{C}\right.$ for $45 \mathrm{~s}$, $61^{\circ} \mathrm{C}$ for $45 \mathrm{~s}, 72^{\circ} \mathrm{C}$ for $45 \mathrm{~s}$ ). In the case of $\beta$-actin, an annealing temperature of $60^{\circ} \mathrm{C}$ was used. Amplified PCR products were resolved in $1 \%$ agarose gel electrophoresis. Negative controls were provided by omitting reverse transcriptase from the cDNA synthesis reaction.

\subsection{Quantitative analyses}

The estimations were conducted by taking into account all the atretic follicles in 384 sections examined. The total of granulosas cells were counted per each follicle. The intensity of label to Beclin-1 and LC3 immunodetections was measured using the Image Processing and Analysis Java (ImageJ) program[17],from 1200 granulosa cells of different atretic follicles. The background of the slide was evaluated with ImageJ program tools. For comparison of intensity of fluoresce between the normal and altered autophagy process, one way ANOVA was conducted with a $\mathrm{P}<0.05$.

\section{Results}

To define the morphological characteristics of the atretic follicles in rat ovaries, we made an examination by a light and electron microscope of ovary sections.

The initial features of the process of cell death in granulosa cells include the contraction of cell volume and the separation among them with the loss of most cell-to-cell contacts. As contraction progresses, the remaining cell contacts take place between prolongations of neighboring follicular cells that cross a large intercellular space. A greatly reduced number of prolongations of the follicular cells penetrate the pellucida to make contact with the cell membrane of the oocyte. In the figure $1 \mathrm{~b}$, an arrow points to one of these remaining prolongations. Numerous granulosa cells contain clear cytoplasmic vacuoles, indicating the 
onset of an autophagic process of cell death (Fig.1b). In the normal follicles the granulosa cells are bonded together as is shown in the Fig. 1a.
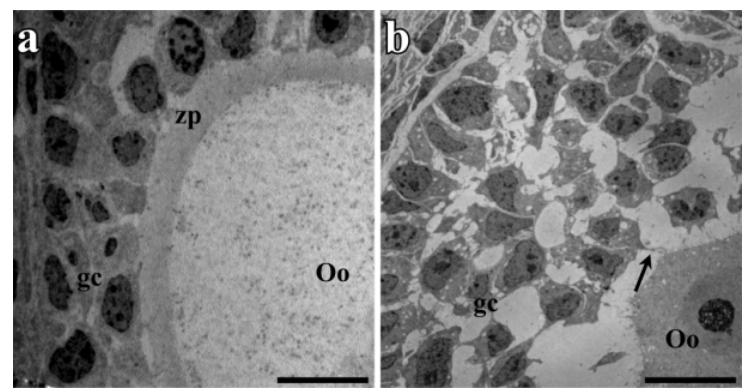

Figure 1. Growing follicles. (a) Is showing a normal follicle. (b) Follicle in process of atresia showing ample abnormal spaces between deformed granulosa cells. Clear vacuoles are frequent in the cytoplasm of these cells. The relationships between granulosa cells and the oocyte are almost completely lost, except for a few prolongations of the former, one of which is indicated by the arrow.Oo-oocyte; gc-granulosa cell; zp-zonapellucida. Scale bar 2 microns.

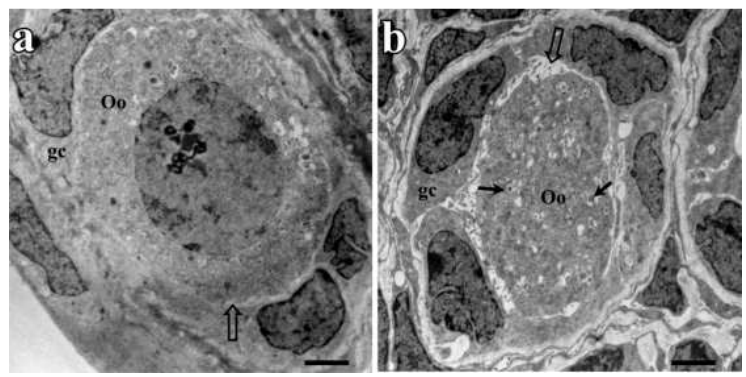

Figure 2. Primordial follicles.a) Non atretic follicle. The granulosa cells are in contact one another and maintain the relationship with the oocyte (empty arrow). The cytoplasm of the oocyte has few autophagosomes, considered as physiological autophagy. (b) The cytoplasm of the oocyte is extensively vacuolated and it is contracted, indicating that it is undergoing a process of autophagic death(empty arrow). However, the granulosa cells are almost normal except for a few clear vacuoles. The arrows point to autophagic vacuoles containing residues of organelles.Oo-oocyte; gcgranulosa cell. Scale bar 2 microns.

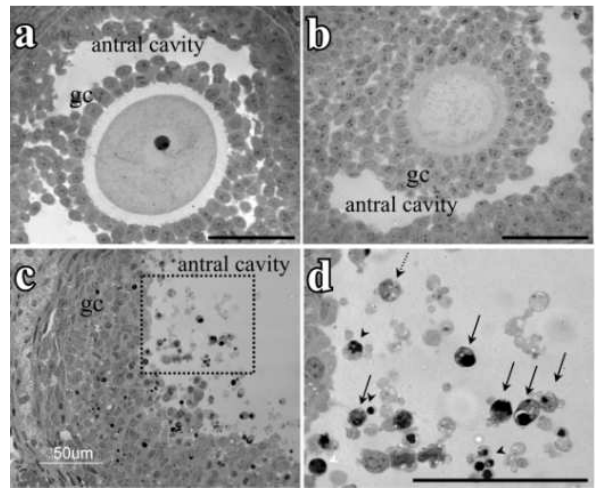

Figure 3. Light microscopic images of antral follicles. (a) and (b) are showing healthy follicles. (c) Antral follicle in an advanced stage of atresia. The cavity of the follicle contains granulosacells in an advanced process of cell death. The rectangle indicates the area enlarged in figure $d$. In (d) the arrows point to cells with dark, compacted nuclei and clear cytoplasmic vacuoles. Arrowheads point to severely altered cells in the last stages of the apoptotic process of cell death. gc-granulosa cells. Scale bars 50 microns.
The atresia of primordial follicles is characterized by morphologically normal granulosa cells and important autophagic alterations of the oocyte in the form of clear vesicles, some of which contain cytoplasmic debris (Fig.2b). These features suggest that the process of atresia is triggered in these follicles by alterations of the oocyte, which are not present in a normal follicle (Fig.2a).

In healthy follicles, the granulosa cells are attached between them, the oocyte is rounded (Figs. $3 \mathrm{a}$ and $3 \mathrm{~b}$ ), and there are no altered cells in the antral cavity. In antral follicles undergoing atresia the granulosa cells suffer processes of cell death that are typical of either apoptosis or autophagy (Fig. 3). Their nuclei have abundant compact chromatin and their cytoplasm is frequently vesiculated (Fig. 3d). Cells in the final stages of the death process lose contacts with other granulosa cells and undergo fragmentation of the cytoplasm through budding; however there is no apparent destruction of the cell membrane. Both nuclei without cytoplasm and portions of cytoplasm without nuclei can be found in the antral cavity (Fig.3d).

The ultrastructural evaluation of granulosa cells in atretic follicles evidenced several alterations, including the classical apoptotic morphology (Fig.4a); elevated amount of autophagic vacuoles in the cytoplasm. However, their nuclei are notapparentlyhighly altered (Fig.4b). High magnification of the autophagic cell cytoplasm allowsappreciating vesicles with cytoplasmic content in different degree of degradation (Fig.4c). A third type of ultrastructuralalterations was evidenced in cells with simultaneous characteristics of apoptosis and autophagy, since they have highly compact chromatin corresponding to apoptosis cell death and anumerous autophagic vesicles (Fig.4d). Our ultrastructural observations indicate that the granulosa cells of atreticfollicles are dying with morphological characteristics corresponding to apoptosis, autophagy and with simultaneous characteristics of both cell death processes.

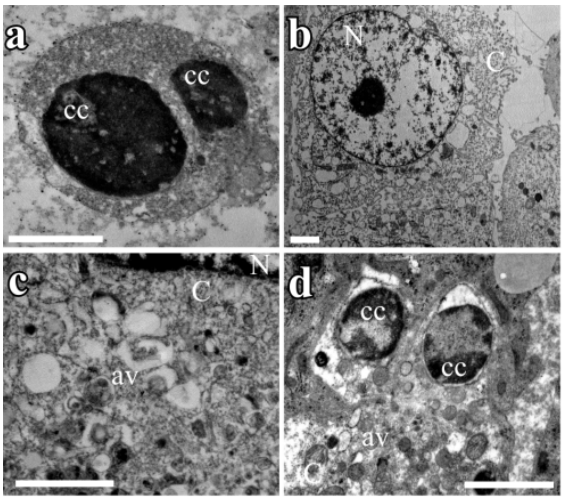

Figure 4. Electron microscopy views of altered granulosa cells. Classical apoptotic characteristics as compact chromatin are present (a). Numerous vesicles are present in several granulosa cells (b). High magnification of the cytoplasm shows the autophagic vesicles (c). Some granulosa cells with ultrastructural characteristics of apoptosis and autophagy (d). cccompact chromatin; C-cytoplasm; $N$-nucleus; av-autophagic vesicle. Scale bars 2 microns. 


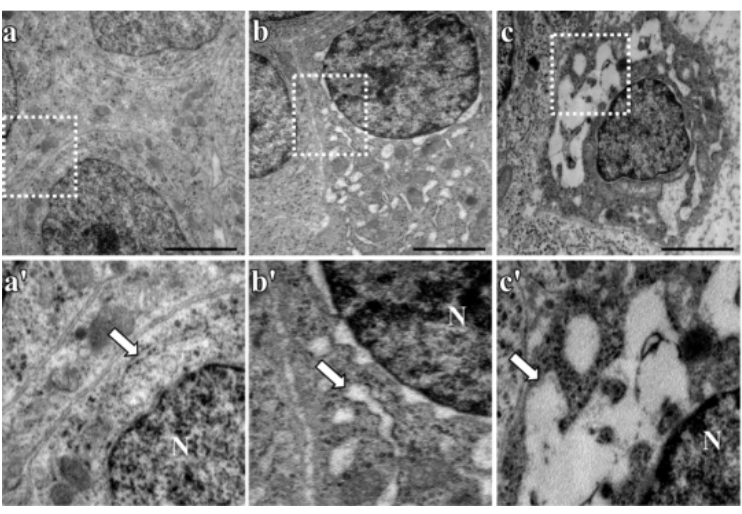

Figure 5. Granulosa cells of follicles during the process of atresia. (a) Normal granulosacellswith normal endoplasmic reticulumas can be seen in high magnification in $a$ '. In some granulosa cells the lumen of the nuclear envelope and the cisterns of the endoplasmic reticulum are extremely swollen ( $b, b^{\prime}, c$ and $c$ '). Other neighboring follicular cells appear to be almost normal. Arrows point the endoplasmic reticulum. $\mathrm{N}$-nucleus. Scale bars 2 microns.

Some altered cells showed swelling of the lumen of the nuclear envelope and of the cisterns of the endoplasmic reticulum. These cells co-exist with normal granulosa cells and other follicular cells that are undergoing distinct processes of cell death (Figs.5b and $5 \mathrm{c}$ ). These alterations are not present in the normal cells (Fig.5a).

To corroborate our structural and ultrastructural observations we immunodetected specific proteins of apoptosis and autophagy cell death in the same cell. To identify autophagy we have immunodetectedBeclin-1 and LC3 proteins. We used the active-caspase- 3 immunolocalization, and TUNEL technique to evaluate the apoptosis.

Taken in account that the autophagy is a physiological process, the basal autophagic process was discriminated from autophagic cell death, measuring the fluorescence intensity corresponding to the Beclin-1 and LC3 immunodetections (graphs 1a and 1b). Statistical analyses showed that the fluorescence signals between the two groups were significantly different.

\section{a}

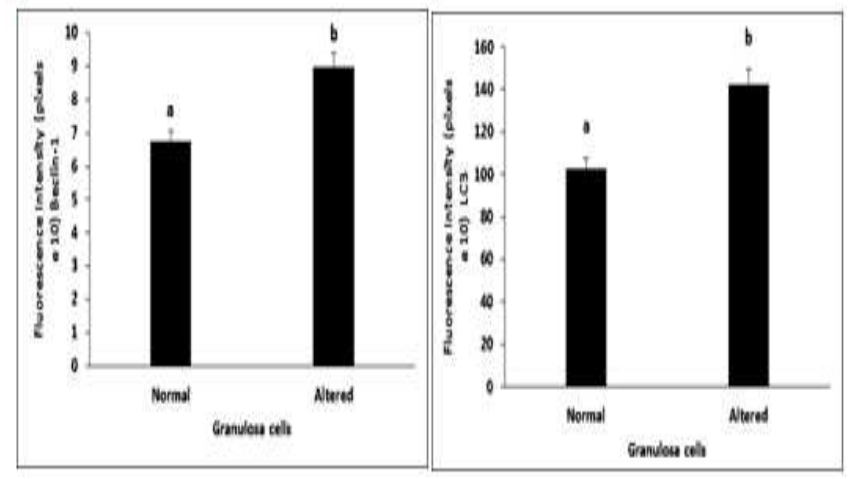

Graph 1 Quantitation of fluorescence. (a) Beclin-1immunodetection. (b) LC3 immunodetection. The intensity of labeling in the altered granulosa cells was significantly different from that in the normal ones. Standard error is represented in each bar. $a$ and $b$ are significantly different $(P<0.05$, ANOVA; $n=1200)$.

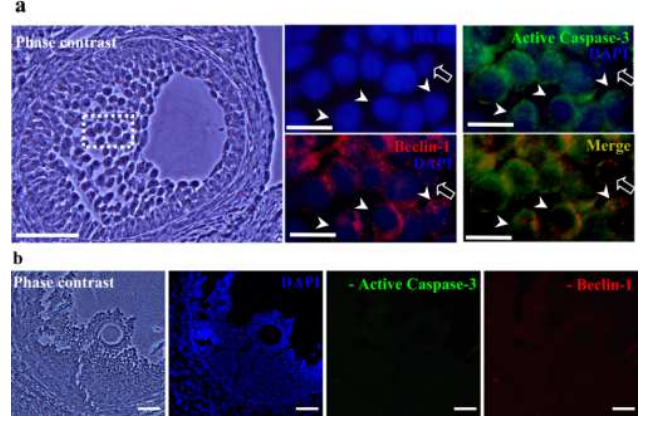

Figure 6. Light microscopic images of atretic follicles. (a) Antralfollicle. The phase contrast image shows thegeneral morphology of the follicle. The granulosa cells are detached from their neighbors. The dotted square region is the zone shown at a higher magnification. DAPI is evidencing the chromatin disposition. Some cells are positive to active caspase-3 (in green). Some of these cells are also positive to Beclin-1 (in red, empty arrow). Active caspase- 3 and Beclin-1 are simultaneously present in few granulosa cells (arrow heads), as the merging of the images reveals. (b) Atretic follicle with antrum as the negative control to active caspase-3 and Beclin-1 immunodetections. Scale bars 20 microns.
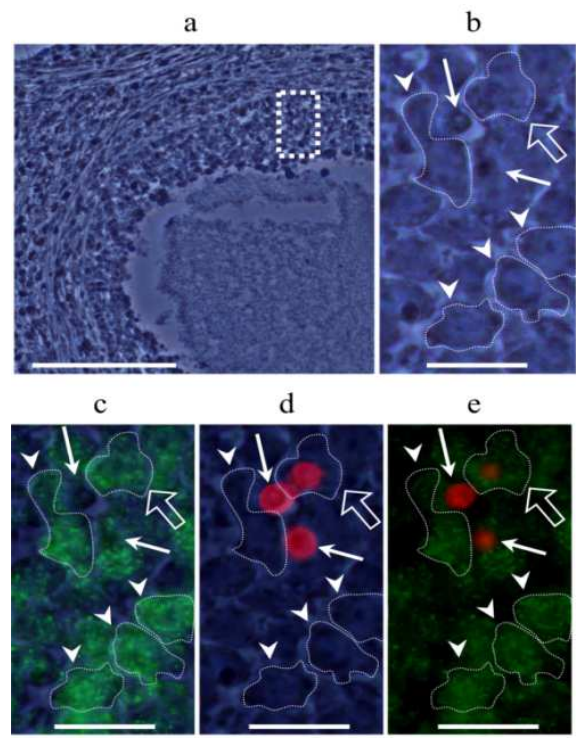

Figure7.Phase contrast image showing the morphology of granulosa cells in a large antral follicle in an advanced process of atresia (a), the dotted square is the zone shown at high magnification. Some cellular shapes evidenced by the phase contrast are delineated by dotted lines (b). The arrow heads point to granulosa cells that are positive only for the autophagic marker LC3 (c). Some contracted cells that are positive only for the apoptotic marker TUNEL (arrows in d). The empty arrow points to one cell positive for autophagic and apoptotic markers; LC3 (green) and TUNEL (red) respectively (e). Scale bars (a) 100 microns; all the others 10 microns.

Two simultaneous light microscope immunolocalizations of active caspase-3 and Beclin-1, as LC-3 and TUNEL technique were carried out to localizeapoptosis and autophagy in the same cells. The results reveal that somegranulosa cells of follicles in the process of atresia are simultaneously positive for both markers. Figure6 shows the presence of active caspase-3 (apoptosis) and Beclin-1 (autophagy) in a section of anantral follicle. Some cells are only positive to active caspase- 3 or to Beclin-1, and simultaneous positive cells were found to a lesser extent (high 
magnifications Fig.6). Simultaneous LC3 and TUNEL detection showed that some cells are positive to the autophagic marker LC3 (Fig. 7c); other cells are positive to the TUNEL technique (Fig.7d), and few of them are positive to both markers (Fig.7e). These findings indicate that proteins of both processes of cell death are already present in follicles when the process of cell deathis carried out.

Markers of apoptosis and autophagy are not always present to the same extent in all granulosa cells. Most of those cells are positive to markers of apoptosisor autophagy, and few are double positive to both apoptosis and autophagy (table 1).

Table 1. Percentage of granulosa cells positive to the different proteins to identify apoptosis and autophagy processes; $n=11,188$.

\begin{tabular}{cccc}
\hline label & $\begin{array}{c}\text { \% posi- } \\
\text { tive } \\
\text { granulosa } \\
\text { cells }\end{array}$ & label & $\begin{array}{c}\text { \% posi- } \\
\text { tive } \\
\text { granulosa } \\
\text { cells }\end{array}$ \\
\hline Active Caspase-3 & 67.29 & TUNEL & 8.54 \\
Beclin-1 & 0.61 & LC3 & 35.99 \\
Act Casp-3+Beclin-1 & 32.08 & TUNEL+LC3 & 4.30 \\
\hline
\end{tabular}

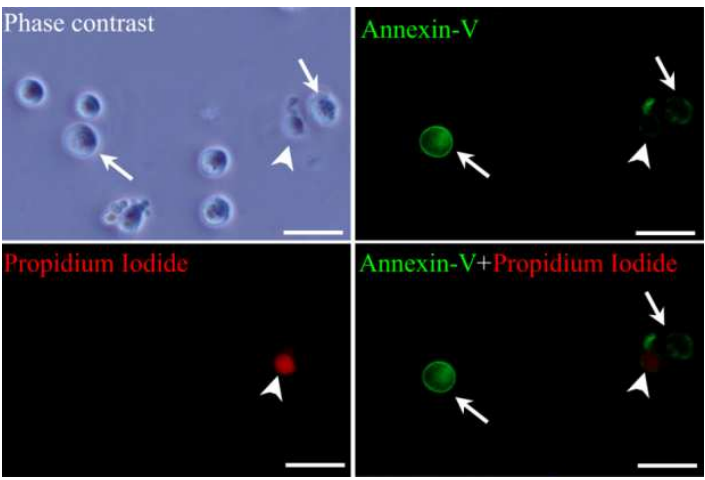

Figure 8.Annexin-V assay in granulosa cells. The phase contrast image shows the general morphology. The granulosa cells positive to the Annexin-V incorporation (arrows) and negative to propidium iodide were apoptotic cells; the cells positive to both markers were considered as necrotic (arrow head). There were cells negative to both marks, considered as healthy. Scale bar 20 microns.

The tendency and variation of the percentage of the markers to apoptosis and autophagy cell death were similar in each individual analyzed.

The apoptosiswas evaluated in isolated cells by means of the Annexin-V labeled with FITC incorporation, which binds to the phosphatidylserine located on the external side of the cell membrane of the isolated cells. Supra-vital propidium iodide labeling was used to identify dying cells with damaged cell membrane. Our results showed that the granulosa cells are positive to the Annexin-V incorporation (Fig. 8), evidencing the apoptosis process.

After analyzing these ultrastructural and immunohistochemical characteristics, we evaluated the molecular and biochemical properties of granulosa cell populations. The mRNA corresponding to the pro-apoptotic caspase- 3 and pro-autophagic LC3 genes was evaluated via the RT-PCR technique. Results indicate the expression of both genes in the same granulosa cell population (Fig. 9a).

The mature, functional stage of the proteins corresponding to the mRNA of LC3 and caspase-3 was evaluated with Western Blot. The level of autophagy was assessed by detecting the conversion of LC3-I to LC3-II, which correlates with the amount of autophagosomes in follicular cells. To investigate the participation of apoptosis, we assessed the presence of active caspase- 3 in the granulosa cells. Results suggest that the active form of caspase- 3 is present in follicular cells (Fig. 9a).

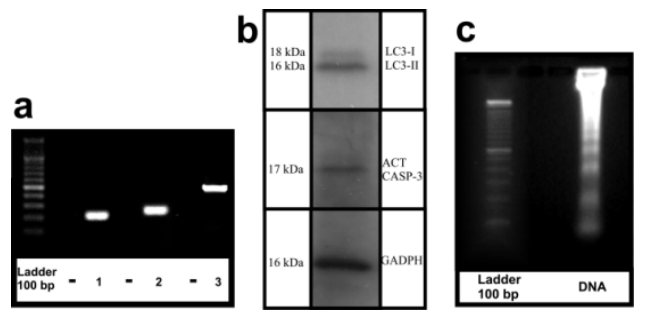

Figure 9.(a) RT-PCR to identify mRNA from caspase-3 (1), LC3 (2) and $\beta$-actin (3). The negative (-) control was developed by omitting the reverse transcriptase from the cDNA synthesis reaction. (b) Western Blot for LC3, active caspase-3 and GADPH proteins. Lysates from granulosa cell populations indicate the protein bands that correspond to the two LC3 forms. Bands are detected at 18 and $16 \mathrm{kDa}$ and correspond to membrane-bound LC3-I and LC3-II, respectively. Active caspase-3 is detected at $17 \mathrm{kDa}$. GADPH protein is detected at $16 \mathrm{kDa}$. (c) Agarose gel electrophoresis of DNA extracted from granulosa cells, showing apoptotic DNA fragmentation in this cellular population.

Finally, we evaluated the fragmented DNA using agarose gel electrophoresis, which showed the typical DNA ladder pattern of apoptosis in the granulosa cell population (Fig. 9c).

\section{Discussion}

Apoptosis and autophagy are two programmed cell death events involved in tissue homeostasis and development. In follicular atresia, cell death plays an important role in maintaining normal follicular maturation. Studies of follicular atresia in several species have shown that granulosa cells display typical traits of apoptosis, such as nuclei with chromatin margination, pyknotic nuclei, fragmented DNA, and apoptotic bodies[reviewed in 18]. Apoptotic cell death was found to occur simultaneously with mitosis within the same follicle; a result consistent with the notion that atresia is determined by a dynamic equilibrium between cell division and cell death [19].Conventionally, the death of granulosa cells in mammalian ovaries during the process of follicular atresia was thought to be apoptotic in nature; however, morphological characteristics of other forms of cell death have been seen in birds [20].Observations from the present study demonstrate that granulosa cells from mammalian ovaries die through a variety of processes; i.e., both programmed cell death processes apoptosis and autophagy. 
The apoptotic morphology includes cellular contraction, chromatin condensation [21]. The main biochemical characteristics of apoptosis are the activation of cysteineaspartic acid-specific proteases (caspases) [22; 23; 24] and DNA fragmentation [21], which are processes possible to identify by several techniques as TdTdUTP nick end labeling (TUNEL) assay [25], DNA agarose gel electrophoresis $[26,27]$, and by the loss of the cytoplasmic membrane asymmetry with the phosphatidylserine externalization [28]. In this work we use morphological and biochemical criteria to characterize the apoptotic cells.

Autophagy is involved in physiological processes as recycling intracellular compounds, in a cytoprotective manner when occurs at basal levels [reviewed in 29]. When autophagic process increases, the cell may be engaged in an irreversible process of death with autophagic features [30]. There are several strategies to discriminate the basal levels of autophagy from the increased autophagy conducting to the cell death [31]. In the present study we include the evaluation of the amount of autophagosomes by means of transmission electron microscopy, the presence of Atg proteins (as Beclin-1 and LC3) by means of immunofluorescence, and the LC3 lipidation revealed by the Western blot technique [reviewed in 32]. It is important to mention that to define the autophagy cell death, we have taken into account morphological and biochemical characteristics of the altered cells. Furthermore, we could distinguish autophagy from apoptosis, by means of their different biochemical and morphological characteristics.

To assert that both processes of cell death, apoptosis and autophagy are present in a granulosa cell it was necessary to identify morphological and/or biochemical characteristics of both processes in the same cell. It means that the altered cells were simultaneously positive to Beclin-1 and active caspase-3, LC3 and TUNEL or that the ultrastructural features of these cells showed an exacerbated amount of autophagic vesicles and a highly compacted nucleus.

Normal granulosa cells are connected by gap junctions that facilitate the transfer of nutrients, growth factors and oxygen between them [reviewed in 33]. Our structural and ultrastructural observations show that in some follicles the first signs of the alteration of granulosa cells are the contraction of cytoplasm, changes in shape, and the partial or total loss of contact among them. All these characteristics correspond to the apoptosis process, and the localization of pro-apoptotic factors such as active caspase-3, DNA fragmentation as identified by the TUNEL technique, andAnnexin-V incorporation, supporting the idea that a significant number of cells death by apoptosis. However, the ultrastructural alterations observed in the present work, show that not only apoptotic features exists in dying granulose cells, there are also a high quantity of autophagic vesicles in the granulosa cells. These findings are coincident with the increased localization of the pro-autophagic Beclin-1 and LC3 proteins in the granulosacells undergoing cell death. We have speculated that theextreme restriction of the flow of nutrients as consequence of the loss of contact between the granulosa cells in the process of cell death,couldinduce the over expression of pro-autophagic proteins, such as Beclin-1 and LC3 in granulosa cells during the atresiaprocess. This continuous limitation of nutrients provokes a high expression of these pro-autophagic proteins directingthe cells toward an autophagic cell death, which is evidenced by numerous autophagosomes in the cytoplasm of the granulosa cells observed with the electron microscopy.

Additionally, we found ultrastructural characteristics of both types of cell death in the same cell as highly compact chromatin corresponding to a hallmark of apoptosis with combination with numerous autophagic vesicles. The immunohistochemical analyses showed double positive cells to active caspase-3 and to Beclin-1 proteins, as TUNEL and LC3 markers, demonstrating that although to a lesser extent this alternative rout of cellular elimination is present in granulosa cells of atreticfollicles.All these patterns of cell death process were observed along the different follicular developmental stages.

Our morphological and immunohistochemicalobservations were further strengthened by biochemical analyses and molecular assays, which showed that the mRNAs of the caspase-3 and LC3 areexpressed in isolated granulosa cell populations. Moreover, the expression of active caspase-3 and LC3 proteins during follicular atresia, evaluated by Western Blot, further confirms our morphological findings. In addition, the conversion of LC3 to the lapidated form (LC3-II) proved the presence of autophagy in the granulosa cells. Finally, DNA fragmentation observed in theagarose gel electrophoresis of DNA extracted fromgranulosa cells of rat ovaries corroborates the TUNEL observations, as the apoptotic bodies observed at ultrastructural level. Choi et al. have observed in a modified model, that the induction of autophagy in granulosa cells triggers the initiation of apoptosis; suggesting that both processes are involved in regulating granulosa cell death [34]. It is interesting to note that the present work was realized in intact animals under physiological environmental, without artificial stimulation, thus our results indicate the prevalence of several routes of cell death during the follicular atresia in the rat ovary.

Our results show that the morphological and biochemical characteristics of apoptosis and autophagy are present during granulosa cell death, and they exclude the necrosis process, since we did not find ultrastructural evidences of breakdown of cytoplasmic membrane or degraded DNA in the agarose gel electrophoresis. The Annexin-V assay demonstrated the membrane integrity in cells negative to propidium iodide and positive to the phosphatidylserine externalization. All these results are indicative that necrosis does not participate in follicular atresia.

We observed images of a process of granulosa cell death under electron microscopy that involve the dilatation of the cisterns of the endoplasmic reticulum and the nuclear 
envelope. No other alterations, such as those seen in apoptosis or autophagy, were found in these cells, suggesting that granulosa cells may dye by a process not found in other tissues.

Autophagic cell death is a multi-step process regulated by various signaling pathways that can be activated when the ER is under stress conditions [35]. However, it is also well known that apoptosis can be activated via ER stress, which ends with activation of the apoptotic machinery [36]. Theseultrastructural alterations of granulosa cells observed in this study may be explained by the presence of proautophagy and pro-apoptotic proteins in the same cell, since both types of cell death -apoptosis and autophagycould be initiated by a step that entails ER alterations. Thus, our results suggest that both mechanisms participate in cellular elimination, as the double immunolocalizations also show.

The present work demonstrates the participation of proteins belonging to apoptosis and to autophagy in the same cell or in different cells during the follicular atresia. However, it was not possible to define which of both processes of cell death is activated in first place, because our observations were carried out in an in situ environment which image is an instant of the cell life. On the other hand, these procedures allowed us to obtain ultrastructural evidences of processes of cell death not found in other cell types, as a large increase of the lumen of nuclear envelope, and of the cisterns of the endoplasmic reticulum. However, it is necessary a study carried out with different methods to evaluate a possible sequence of different stages of the processes of cell death.

\section{Conclusions}

Our observations demonstrate that granulosa cells decay not only by means of the typical processes of apoptosis or autophagy, but also through alterations not found in other organs. All our observations lead us to propose that during follicular atresia in rat ovaries cell death occurs through a combination of processes that involves apoptosis and autophagic cell death, a mixed event that includes both routes in the same cell and also by processes not found in other tissues.

\section{Acknowledgments}

This work was supported by the grants CONACYT 180526 and PAPIIT IN212912. The authors would like to thank Ernestina Ubaldo Pérez for technical assistance. They also thank Paul C. Kersey Johnson for reviewing the English word usage and grammar.

\section{References}

[1] Jiang J.Y., ChengC.K., WangY., TsangB.K. Regulation of cell death and cell survival gene expression during ovarian follicular development and atresia. Front Biosci.,2003;8:d222-237.

[2]Morita Y., Tilly J.L. Oocyte apoptosis: like sand through an hourglass Dev Biol.,1999; 213(1):1-17.

[3] Ortiz R., Echeverría O.M., Salgado R., Escobar M.L., Vázquez-Nin G.H. Fine structural and cytochemical analysis of the processes of cell death of oocytes in atretic follicles in new born and prepubertal rats. Apoptosis,2006; 11:25-37.

[4]Escobar M.L., Echeverría O.M., Vázquez-Nin G.H. Immunohistochemical and ultrastructural visualization of different routes of oocyte elimination in adult rats. Eur J Histochem.,2012; 56(2):102-109.

[5] Escobar M.L., Echeverría O.M., Sánchez-Sánchez L., Méndez C., Pedernera E., Vázquez-Nin G.H. Analysis of different cell death processes of prepubertal rat oocytes in vitro. Apoptosis, 2010; 15(4): 511-526.

[6] Escobar M.L., Echeverría O.M., Ortiz R., Vázquez-Nin G.H. Combined apoptosis and autophagy, the process that eliminates the oocyte of atretic follicles in immature rats. Apoptosis, 2008; 13(10):1253-1266.

[7] Tilly J.L., TillyK.I.,PerezG.I. The genes of cell death and cellular susceptibility to apoptosis in the ovary: a hypothesis. Cell Death Differ., 1997; 4(3):180-187.

[8] Thornberry N.A., LazebnikY.Caspases: enemies within. Science, 1998; 281:1321-1316.

[9] Tsujimoto Y., Shimizu S. Another way to die: autophagic programmed cell death. Cell Death Differ.,2005;12 Suppl $2: 1528-1534$

[10] Straub M., BredschneiderM., ThummM. AUT3, a serine/threonine kinase gene, is essential for autophagocytosis in Saccharomyces cerevisiae. J Bacteriol.,1997;179:38753883 .

[11] Holt S.V., WyspianskaB.,RandallK.J., JamesD., FosterJ.R., WilkinsonR.W. The development of an immunohistochemical method to detect the autophagy-associated protein LC3-II in human tumor xenografts. ToxicolPathol.,2011;39:516-523.

[12]Klionsky D.J., EmrS.D. Autophagy as a regulated pathway of cellular degradation. Science,2000;290(5497):1717-1721.

[13] He C., Levine B. The Beclin 1 interactome.Curr Op Cell Biol. $2010 ; 22: 140-149$.

[14] Kang R., Zeh H.J., Lotze M.T., Tang D. The Beclin 1 network regulates autophagy and apoptosis. Cell Death Differ. $2011 ; 18: 571-580$.

[15] Takahashi Y., Coppola D., Matsushita N., Cualing H.D., Sun M., Sato Y., Liang C., Jung J.U., Cheng J.Q., Mulé J.J., Pledger W.J., Wang H.G. Bif interacts with Beclin 1 through UVRAG and regulates autophagy and tumorigenesis. Nat Cell Biol. 2007; 9:1142-1151.

[16] Boya P., González-Polo R.A., Casares N., Perfettini J.L., Dessen P., Larochette N., Métivier D., Meley D., Souquere S., Yoshimori T., Pierron G., Codogno P., Kroemer G. Inhibition of macroautophagy triggers apoptosis. Mol. Cell. Biol. 2005; 25:1025-1040. 
[17] Abramoff M.D., MagalhaesP.J., RamS.J. Image processing with ImageJ. BiophotonicsInternational,2004; 11:36-42.

[18] Hussen M.R. Apoptosis in the ovary: molecular mechanisms.Human Reprod., 2005; 11(2):161-177.

[19]Jolly P.D., SmithP.R., HeathD.A., HudsonN.L., LunS., StillL.A., WattsC.H., McNattyK.P. Morphological evidence of apoptosis and the prevalence of apoptotic versus mitotic cells in the membrane granulosa of ovarian folGalluzlicles during spontaneous and induced atresia in ewes. BiolReprod.,1997;56:837-846.

[20]D'Herde K., De Prest B., Roels F. Subtypes of active cell death in the granulosa of ovarian atretic follicles in the quail (Coturnixcoturnix japonica). ReprodNutr Dev., 1996;36(2):175-189.

[21] Kerr J.F., Wyllie A.H., Currie A.B. Apoptosis. A basic biological phenomenon with wide-ranging implications in tissue kinetics.1972; Br J Cancer. 26:239-257.

[22] Kaufmann S. H., Desnoyers S., Ottaviano Y., Davidson N. E., Poirier G. G. Specific proteolytic cleavage of poly(ADPribose) polymerase: an early marker of chemotherapyinduced apoptosis. 1993. Cancer Res. 53(17):3976-85.

[23] Alnemri E. S., Livingston D. J., Nicholson D. W., Salvesen G., Thornberry N. A., Wong W. W., Yuan J. Human ICE/CED-3 protease nomenclature. Cell. 1996; 87(2):171.

[24] Nicholson D.W., Melino G. Caspases and Cell Death.Encyclopedia of Biological Chemistry.2013; 388-396.

[25] Gavrieli Y., Sherman Y., Ben-Sasson S. A. Identification of programmed cell death in situ via specific labeling of nuclear DNA fragmentation. 1992. J Cell Biol. 119(3):493-501.

[26] Arends MJ, Morris RG, Wyllie AH.Apoptosis.The role of endonuclease.Am J Pathol. 1990;136:593-608.

[27] Gong J. P., Traganos F., Darzynkiewicz Z. A selective procedure for DNA extraction from apoptotic cells applicable for gel electrophoresis and flow cytometry. Anal Biochem. 1994;218:314-319.

[28] Fadok V. A., Voelker D. R., Campbell P. A., Cohen J. J., Bratton D. L., Henson P. M. Exposure of phosphatidylserine on the surface of apoptotic lymphocytes triggers specific recognition and removal by macrophages. J Immunol. 1992; 148(7):2207-16

[29] Levine, B., Kroemer, G. Autophagy in the pathogenesis of disease. Cell, 2008; 132:27-42.

[30]Levine B, Yuan J. Autophagy in cell death: an innocent convict?. J Clin Invest., 2005; 115:2679-2688.

[31] Klionsky D, and 1269 others. Guidelines for the use and interpretation of assays for monitoring autophagy. Autophagy, 2012; 8(4):445-544.

[32] Galluzzi L. and 78 others. Guidelines for the use and interpretation of assays for monitoring cell death in higher eukaryotes. Cell Death Differ. 2009 Aug;16(8):1093-107.

[33] van den Hurk R., Zhao J. Formation of mammalian oocytes and their growth, differentiation and maturation within ovarian follicles Theriogenology,2005; 63:1717-1751.

[34] Choi J., JoM., ChoiD. Induction of apoptotic cell death via accumulation of autophagosomes in rat granulosacells.FertSteril.,2011; 95:1482-1486.

[35]Høyer-Hansen M., BastholmL., SzyniarowskiP., CampanellaM., SzabadkaiG., FarkasT., BianchiK., FehrenbacherN., EllingF., RizzutoR., MathiasenI.S., JäätteläM. Control of macroautophagy by calcium, calmodulin-dependent kinase kinase-beta, and Bcl-2. MolCell.,2007;25(2):193-205.

[36]Oakes S.A., Opferman J.T., Pozzan T., Korsmeyer S.J., Scorrano L. Regulation of endoplasmic reticulum $\mathrm{Ca} 2+\mathrm{dy}-$ namics by proapoptotic BCL-2 family members.BiochemPharmacol.,2003;66(8):1335-1340. 